Research Paper

\title{
Microcystin-LR (MCLR) Induces a Compensation of PP2A Activity Mediated by $\alpha 4$ Protein in HEK293 Cells
}

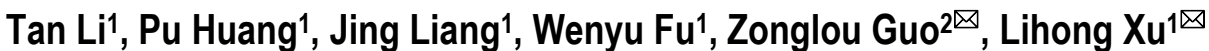

1. Department of Biochemistry and Genetics, School of Medicine, Zhejiang University, Hangzhou 310058, China

2. Department of Biosystem Engineering, College of Biosystem Engineering and Food Science, Zhejiang University, Hangzhou 310058, China

Corresponding author: Zong-Lou Guo and Li-Hong Xu, 866th Yu Hang Tang Road, Hangzhou 310058, China. Fax: 86-571-86967920 and 86-571-88208265; E-mail: zlguo@zju.edu.cn and xulihong@zju.edu.cn

(C) Ivyspring International Publisher. This is an open-access article distributed under the terms of the Creative Commons License (http://creativecommons.org/ licenses/by-nc-nd/3.0/). Reproduction is permitted for personal, noncommercial use, provided that the article is in whole, unmodified, and properly cited.

Received: 2011.03.31; Accepted: 2011.05.30; Published: 2011.06.08

\begin{abstract}
Protein phosphatase 2A (PP2A) is a major protein phosphatase with important cell functions. Known and utilized as a potent inhibitor of PP2A, microcystin-LR (MCLR) targets PP2A as a core element that affects numerous cellular mechanisms. But apart from direct inhibition, the exact effect of MCLR on PP2A in cell is largely unknown, specifically with regard to cellular response and autoregulation. Here, we show that a low concentration of MCLR stimulates, rather than inhibits, PP2A activity in HEK293 cells. Immunoprecipitation and immunofluorescence assays reveal that the catalytic subunit and a regulatory subunit of PP2A, termed a4, dissociate from inactive complex upon MCLR exposure, suggesting that the released catalytic subunit regains activity and thereby compensates the activity loss. At high concentrations of MCLR, PP2A activity decreases along with dissociation of the core enzyme and altered post-translational modification of its catalytic subunit. In addition, the dissociation of $a 4$ and PP2A may contribute to destabilization of HEK293 cells cytoskeleton architecture, detachment to extracellular matrix and further anoikis. Our data provide a novel PP2A upregulation mechanism and challenge the recognition of MCLR only as a PP2A inhibitor in cells.
\end{abstract}

Key words: microcystin-LR; protein phosphatse 2A; a4; anoikis

\section{Introduction}

Microcystin-LR (MCLR) is one of the most toxic secondary metabolites produced by freshwater cyanobacteria. Because of its presence in aquatic blooms, the molecule causes a world-wide environmental problem. MCLR in drinking water is implicated in the development of liver cancer and damage to multiple organs including liver, kidney, intestines and brain [1]. The mechanism of MCLR toxicity has been widely accepted as it inhibits two important protein phosphatases (PPs), PP1 and PP2A, as well as causes oxidative stress, in cell [2-3]. Moreover, proteomic studies by our group and others have found that MCLR and MCRR, another microcystin that is also a potent inhibitor of PPs, comprehensively affect various cellular mechanisms, which are mostly regulated by PP2A [4-6]. Indeed, it is acknowledgeable that the effect of MCLR follows a "MCLR - PPs - cellular mechanism" paradigm. However, cellular autoregulation of PPs in response to MCLR is a missing part and still remains elusive.

Protein phosphatase 2A (PP2A) is a major protein phosphatase in the cell that is involved in diverse cellular processes including RNA transcription, DNA repair, cell proliferation and apoptosis [7-9]. Typical$\mathrm{ly}$, the PP2A holoenzyme is comprised of a $36-\mathrm{kDa}$ catalytic subunit (PP2A/C), a 65-kDa scaffold subunit 
(PP2A/A), and one of a variety of regulatory subunits (PP2A/B). The regulatory subunit binds to the core enzyme $(\mathrm{AC})$ to form the holoenzyme and thus determines the specific functions of PP2A [10]. PP2A activity is mainly regulated by its interacting proteins, its regulatory subunits and the post-translational modification of PP2A/C. PP2A/C is also a target of numerous toxins including MCLR, possibly because it is one of the most evolutionarily conserved enzymes in eukaryotic cells [2]. It is clear so far that the covalent binding of MCLR directly and potently inhibits PP2A/C catalytic activity. But we also previously reported that MCLR upregulated the protein level of $\mathrm{PP} 2 \mathrm{~A} / \mathrm{A}$, which may suggest a more intricate effect of MCLR on PP2A in cell [11]. When considering the post-translational modification of PP2A as well as the interaction among its subunits, the effect of MCLR on PP2A could become even more complicated.

Interestingly, a fraction of PP2A/C was found to form a complex with the $a 4$ protein, the homolog of Tap42 in yeast. The amount of Tap42 associated PP2A/C was predicted to be about $2 \%$ of the whole PP2A/C pool. However, there were only $28 \%$ shared sequence identity between $a 4$ and Tap42, thus the amount of $a 4$ associated PP2A/C was still unknown to our knowledge [12]. It is notable that the complex of $a 4$ and PP2A/C is independent of the A and B subunits, as the binding sites between $a 4$ and PP2A/C partially overlap with that of the PP2A A and C subunits [13]. Classically, the $a 4$ binding form of PP2A is believed to either be inactive or show low phosphatase catalytic activity [14-15]. However, a4 has been reported to have important functions regulating PP2A, as it manages PP2A core enzyme assembly and PP2A/C ubiquitination [12, 16-17]. Moreover, a4 was reported to implement many cellular functions through PP2A, including control of cytoskeleton architecture and anti-anoikis/apoptosis [18-21]. Thereby, when PP2A is under the insult of MCLR, whether a4, the essential PP2A regulator in cell, participates in the cellular regulation of PP2A is an interesting question.

In the present study, we used HEK293 (Human Embryonic Kidney 293) cells because kidney is an important target organ of MCLR; while the nephrotoxicity of MCLR is much less understood than its hepatotoxicity. We tested the PP2A activity as well as other biochemical features of PP2A in HEK293 cells, together with cellular behavior after different concentration of MCLR treatment. Surprisingly, PP2A activity in HEK293 cells was stimulated, not inhibited, by low-concentration MCLR treatment, which may be due to the dissociation of $a 4$ and PP2A/C. Under high-concentration MCLR exposure, PP2A activity decreased along with the disruption of the core enzyme and alternation of post-translational modifications on the $\mathrm{C}$ subunit. Moreover, the dissociation of $a 4$ and PP2A/C is correlated with disruption of cytoskeleton, cell detachment and possible anoikis induced by MCLR, suggesting the consequences after $a 4$ and PP2A/C dissociation.

\section{Materials and Methods}

\section{HEK293 Cell Culture and Treatment}

HEK293 cell line was purchased from Cellbank of Chinese Academy of Sciences (Catalog No. GNHu18). HEK293 Cells were cultured in Dulbecco's modified Eagle's medium supplemented with 10\% FBS, in flasks, dishes, or plates, with suitable inoculum ratio according to the amount of cells needed in one experiment, at $37^{\circ} \mathrm{C}$ in a humidified atmosphere with $5 \% \mathrm{CO}_{2}$. Cells were allowed to attach and grow for $24 \mathrm{~h}$ before the media was replaced with fresh media containing MCLR (Alexis, Switzerland). MCLR was resolved and diluted precisely in order to keep the treatment concentrations accurate. At cell treatment, the control groups of each assay were replaced with fresh media without MCLR. The beginning and ending of MCLR treatment were within the logarithmic phase of cell growth. Prior to any cell lysate preparation, cells were washed twice with ice cold PBS, harvested, and gently rinsed with PBS four times via pelleting in microtubes by centrifugation in order to fully remove MCLR in media.

\section{Western blotting}

The protein levels and post-translational modification of interested proteins were tested using Western blotting. Cell extracts were prepared in lysis buffer (50 mM Tris- $\mathrm{HCl} \mathrm{pH}$ 7.5, $150 \mathrm{mM} \mathrm{NaCl}, 15 \mathrm{mM}$ EDTA, 0.1\% TritonX-100, $1 \mathrm{mM}$ PMSF,) with protease inhibitor complex (Roche) on ice for $30 \mathrm{~min}$. After protein concentration was detected using the Bradford method [22], $60 \mu \mathrm{g}$ of cell extract were loaded on SDS-PAGE for electrophoresis and the gels produced were transferred to nitrocellulose membranes. Primary antibodies applied at $4^{\circ} \mathrm{C}$ overnight were: anti-PP2A/A mouse mAb (Cell Signaling Technology), anti-PP2A/C rabbit mAb (Cell Signaling Technology), anti-Caspase-3 rabbit $\mathrm{mAb}$ (Cell Signaling Technology), anti-p-Y307 PP2A/C rabbit mAb (Abcam), anti-methyl-L309 PP2A/C mouse mAb (Abcam), anti-Mid 1 rabbit poly $\mathrm{Ab}$ (Abcam), anti- $a 4$ mouse $\mathrm{mAb}$ (Upstate), anti-MCLR mouse mAb (Alexis), anti- $\beta$-actin mouse mAb (Santa Cruz), anti-Bcl-2 rabbit $\mathrm{mAb}$ (Santa Cruz), anti-Bad rabbit mAb (Bioworld), and anti-GAPDH mouse mAb (KANGCHEN). The 
concentration recommended by producers was used as were the standard Western blotting procedures. Figures representing three independent assays are shown. Densitometric data were collected by Image J software (National Institutes of Health) for further statistical analysis.

\section{PP2A Activity Assay}

PP2A activity was measured by two methods, both determine the amount of free phosphate by measuring the absorbance of a molybdate: malachite green: phosphate complex. First was with a serine/threonine phosphatase assay kit (Promega). Briefly, cells were collected from 6 well plates and washed before lysis on ice in lysis buffer $(50 \mathrm{mM}$ Tris- $\mathrm{HCl} \mathrm{pH} 7.5,0.05 \%$ Triton X-100, $0.1 \mathrm{mM}$ EDTA, $0.5 \mathrm{mM}$ PMSF, $0.05 \%$-mercaptoethanol, $10 \%$ glycerol) with protease inhibitor complex (Roche) for 40 min, with vortex every $10 \mathrm{~min}$. The cell lysate was filtered through a Sephadex G25 column to remove free phosphate. Protein concentration was determined using the Bradford method. $2.5 \mu \mathrm{g}$ of cell protein were distributed equally among the wells of a 96 well plates with the specific peptide substrate RRA(pT)VA in PP2A-specific reaction buffer $(250 \mathrm{mM}$ imidazole $\mathrm{pH}$ 7.2, $1 \mathrm{mM}$ EGTA, $0.1 \%$-mercaptoethanol, $0.5 \mathrm{mg} / \mathrm{ml}$ BSA) at $37^{\circ} \mathrm{C}$ for $18 \mathrm{~min}$. After incubation, $50 \mu \mathrm{l}$ of molybdate dye/additive mixture was added, and the color was allowed to develop for $20 \mathrm{~min}$. The optical density of the samples was read using a 96-well plate reader at $630-\mathrm{nm}$. All determinations were performed in duplicate, and the absorbance of the reactions was corrected by determining the absorbance of control reactions without phosphoprotein substrate. The amount of phosphate released (pmol) was calculated from a standard curve (0-2000 pmol) and normalized using the controls. The second method was using an antibody against PP2A/C to immunoprecipitate PP2A/C before measuring its activity, specifically [16]. Briefly, 300 $\mu \mathrm{g}$ of total cell lysate from each group was prepared as described above. PP2A/C was precipitated from cell lysate using $3 \mu \mathrm{g}$ of PP2A/C antibody (clone 1D6, Upstate) with rotation for $12 \mathrm{~h}$ at $4^{\circ} \mathrm{C}$, and then $40 \mu \mathrm{l}$ of Protein A agarose beads were added for $2 \mathrm{~h}$ rotation at $4^{\circ} \mathrm{C}$. Noteworthy, this PP2A/C antibody does not block phosphatase activity. PP2A activity was measured by incubating the immunoprecipitated protein with the substrate RRA(pT)VA for 5 min at $37^{\circ} \mathrm{C}$, with 1000 rpm shaking. Afterwards, $25 \mu \mathrm{l}$ of reacted solution was mixed with $100 \mu 1$ molybdate dye/additive in a 96 well plate. The analysis of yielded free phosphate from each group was as previously described. Finally, the phosphatase activity was normalized to the relative amount of immunoprecipitated PP2A/C referring to the Western blotting quantification analysis. Data were from three independent assays of each method.

\section{Immunoprecipitation}

Immunoprecipitation was used to test the interaction between interested proteins in this study. Cells were collected from dishes before lysed in RIPA buffer (Millipore) with $0.05 \% \beta$-mercaptoethanol, 1 $\mathrm{mM}$ PMSF and protease inhibitor complex (Roche) at $4^{\circ} \mathrm{C}$ for $40 \mathrm{~min}$. After $14000 \times \mathrm{g}$ centrifugation for 20 min to precipitate the pellet, $\sim 1 \mathrm{mg}$ cell lysate from each group was incubated, with rotation, with $10 \mu \mathrm{l}$ anti-PP2A/C rabbit monoclonal antibody (Cell Signaling Technology) at $4^{\circ} \mathrm{C}$ overnight. $100 \mu \mathrm{l}$ Protein A agarose beads (Santa Cruz) were added and the mixture was rotated for an additional $3 \mathrm{~h}$. Beads were washed four times with RIPA buffer and once with PBS, resuspended in SDS loading buffer and boiled prior to Western blotting. Three selected independent assays are shown.

\section{Immunofluorescence}

Immunofluorescence was used in this study to investigate the localization of interested proteins. About $50 \%$ to $60 \%$ confluent cells on coverslips were washed by PBS, fixed with $4 \%$ formaldehyde $10 \mathrm{~min}$ and blocked with 10\% FBS/PBS (v/v) for $20 \mathrm{~min}$. Cells were then incubated with following primary antibodies: anti-PP2A/C rabbit mAb (Cell Signaling Technology), anti-MCLR mouse mAb (Alexis), anti-a4 mouse mAb (Upstate), anti-vinculin mouse mAb (Abcam). Solutions were diluted to the recommended concentration in $0.1 \%$ saponin/FBS/PBS $(\mathrm{w} / \mathrm{v})$ at $4^{\circ} \mathrm{C}$ overnight. After washing with $10 \%$ FBS/PBS, secondary antibodies of Alexa Fluor 488 goat anti-rabbit IgG conjugate and Alexa Fluor 549 goat anti-mouse IgG conjugate (Jackson ImmunoResearch Laboratories) were applied. Nuclei and F-actin were visualized by staining with DAPI (Sigma) and Phallotoxins (Molecular Probes). Images were captured on a Zeiss microscope (LSM 510 META); figures representing 10 randomly chosen fields are shown.

\section{Cell Detachment Assay}

Cell detachment assay was applied in this study to measure the detachment of cells after MCLR treatment. Equal amounts of cells were seeded onto 12-well plates. Each experimental group, which would further be washed by PBS, corresponded to a control group. After treatment, cells in control groups were trypsinized and harvested in order to measure the total amount of cells. Cells in experimental groups were washed three times in PBS at room temperature 
and shaken gently for $10 \mathrm{~s}$. Adherent cells were then trypsinized and harvested. All cell samples were counted with a Beckman Coulter cell counter. The extent of cell detachment is defined as (the number of control group - the number of experimental group) / (the number of control group). In brief, cell detachment was quantified by counting only adherent cells [23]. Data were from three independent assays.

\section{MTT assay}

MTT assay was used to test the changes of cellular viability after MCLR treatment. HEK293 cells were seeded at equal number in 24 well plates. After treatment, media ware replaced with fresh media containing $0.5 \mathrm{mg} / \mathrm{ml} 3$ - (4,5-dimethylthiazol-2-yl)2,5-diphenyltetrazolium bromide (MTT), and further incubated for $4 \mathrm{~h}$. Following aspirating the culture medium, the resulting formazan was dissolved with dimethylsulfoxide. Plates were placed on a shaker for $10 \mathrm{~min}$ and read immediately at $490 \mathrm{~nm}$ with a microplate reader. Data were from three independent assays.

\section{Statistical analysis}

All data are presented as the mean \pm SD from three independent experiments $(n=3)$. Pairwise comparisons between means were tested by a Student's t-test, two-tailed analysis. n.s. not significant; ${ }^{*}, \mathrm{p}<0.05$; ${ }^{* *}, \mathrm{p}<0.01 ;$ and ${ }^{* * *}, \mathrm{p}<0.001$.

\section{Results}

\section{MCLR exists in HEK293 cells associated with PP2A C subunit}

Recent studies report that a group of hepatocyte uptake transporters termed organic anion transporting polypeptides (OATPs) are crucial for MCLR transfer into cells, and question the presence of MCLR in non-OATP cells [24-25]. To our knowledge, few immortalized kidney cell line expresses OATP, not HEK293 cells as well. Therefore as premise, we first verified the presence of MCLR in non-transfected HEK293 cells by Western blotting with an antibody against MCLR. HEK293 cells were treated with 0.5 $\mu \mathrm{M}-10 \mu \mathrm{M}$ MCLR for 24 hours, and MCLR was then carefully removed from the cultures as described in Experimental Procedures. As shown in Fig. 1A, the bands of proteins recognized by anti-MCLR antibody showed a dose-dependent increase at the ladder of molecular weight of about $30 \mathrm{kDa}$ to $39 \mathrm{kDa}$, similar to that seen in OATP-positive cells [24]. As the molecular of PP2A/C is about $36 \mathrm{kDa}$, the result possibly suggested the binding of MCLR to PP2A/C. To substantiate the prediction, Western blotting was applied at one membrane using antibodies against MCLR and PP2A/C, each with a different IRDye conjunct secondary antibody. As shown in Fig. 1B, there were up-shift bands about $1 \mathrm{kDa}$ above the $36 \mathrm{kDa}$ band in MCLR-treated groups. Because the molecular weight of MCLR is about 995Da, these bands might suggest the MCLR-conjunct bands of PP2A/C. Notably, these up-shift bands also showed a co-migration with the bands of MCLR, which strongly suggest the binding of MCLR and PP2A/C. Also, there were a portion of bands probed by anti-MCLR antibody that did not overlap with the bands of PP2A/C, suggesting other possible MCLR-bound proteins tested. An immunofluorescence assay (Fig. 1C) probed both PP2A/C and MCLR. Though a small amount of non-specific binding was detected, the merged images show that binding of MCLR and PP2A/C increases in a dose-dependent manner, consistent with the results above. Although pinocytosis rather than active transport may be responsible for MCLR uptake, and the quantity of MCLR in HEK293 cells could be much lower than OATP-positive cells, the presence of MCLR in non-OATP HEK293 cells is clear.

\section{PP2A activity undergoes opposite change with alternations of the protein level and modifica- tion of its subunits}

To measure the effect of MCLR on PP2A in the cell, HEK293 cells were exposed to $1 \mu \mathrm{M}-10 \mu \mathrm{M}$ MCLR for 24 hours before being harvested. The MCLR concentration and incubation time were decided according to previous studies performed by our, and other, groups [26-27].

To our surprise, PP2A activity increased at low concentrations of MCLR. After peaking at a $2 \mu \mathrm{M}$ concentration at a rise of about $30 \%$, PP2A activity decreased in a dose-dependent manner to about $20 \%$ for a $10 \mu \mathrm{M}$ concentration (Fig. 2A), measured by a Promega kit. To confirm this result, we tested PP2A activity at $2 \mu \mathrm{M}$ and $10 \mu \mathrm{M}$ MCLR treatment, and control, by an immunoprecipitation phosphatase assay method. Shown in Fig. 2B, PP2A activity changed consistently to the above data. As shown above, MCLR is present in HEK293 cells through the whole range of concentrations used in the PP2A activity assay. Thus, rather than simply inhibition, the data might suggest a more complicated effect of MCLR on PP2A in HEK293 cells.

We tried to explain the opposing activities of PP2A on the protein level of core enzyme. As shown in Fig. 2C, PP2A/A maintains its protein level through the whole range of MCLR dosing. At the treatment of MCLR, the protein level of PP2A/C did not show a significant change. At a high concentration 
(5 $\mu \mathrm{M}$ and above) of MCLR, a band, which increases in a dose-dependent manner, is found approximately $1 \mathrm{kDa}$ above the target $36 \mathrm{kDa}$. Conversely, the band at $36 \mathrm{KDa}$ displayed a dose-dependent decrease. The result might thereby, reconfirm the existence of MCLR in HEK293 cells. However, it could only explain the decrease in PP2A activity, not the increase at low concentrations of MCLR.

We next investigated the post-translational modification of PP2A/C, because reversible methylation and phosphorylation of its carboxy-terminal tail had been reported to regulate its catalytic activity: phosphorylation of PP2A/C at Tyr307 decreases its catalytic activity and methylation of PP2A/C at Leu309 affects its affinity to regulatory subunits.
Therefore, antibodies against phosphorylation and methylation of PP2A/C at these exact sites were applied via Western blotting. However, even if post-translational modification regulates catalytic activity, the changes in PP2A activity can still only be partially explained by the decrease, rather than the increase. As shown in Fig. 2B, the proportion of phosphorylated PP2A/C slightly increased and methylated PP2A/C decreased at high concentrations ( $5 \mu \mathrm{M}$ and above) of MCLR, while both remained constant in the $2 \mu \mathrm{M}$ MCLR group. In addition, the anti-methylated-PP2A/C antibody almost failed to detect the up-shift bands. Because MCLR modification is more likely to be the reason for the loss of PP2A activity, we further discussed the theory in Discussion.
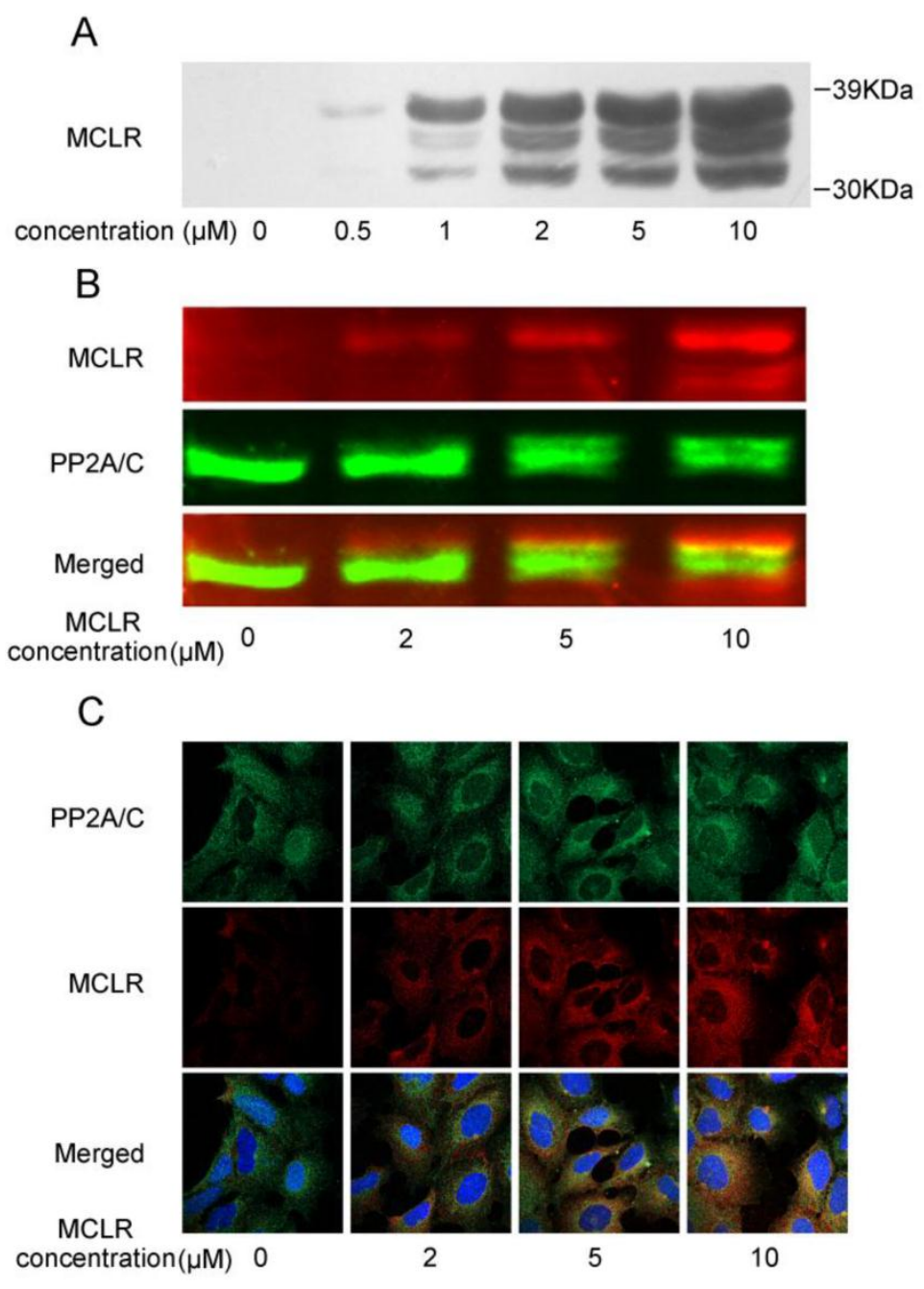

Figure 1. MCLR is associated with the PP2A C subunit in the cell. HEK293 cells were treated with MCLR at varying concentrations for $24 \mathrm{~h}$. (A) Cell lysate was examined by Western blotting with an antibody against MCLR. (B) Cell lysate was tested by Western blotting with antibodies against MCLR (red) and PP2A/C (green) on one nitrocellulose membrane. Each antibody was further probed by IRDye 680 conjugated goat anti-mouse or IRDye $800 \mathrm{CW}$ conjugated goat anti-rabbit secondary antibody (LI-COR) before scanned. Merged imaged showed the co-migration of MCLR and PP2A/C. (C) Immunofluorescence assay of MCLR (red), PP2A/C (green) and nuclei (blue). 
A

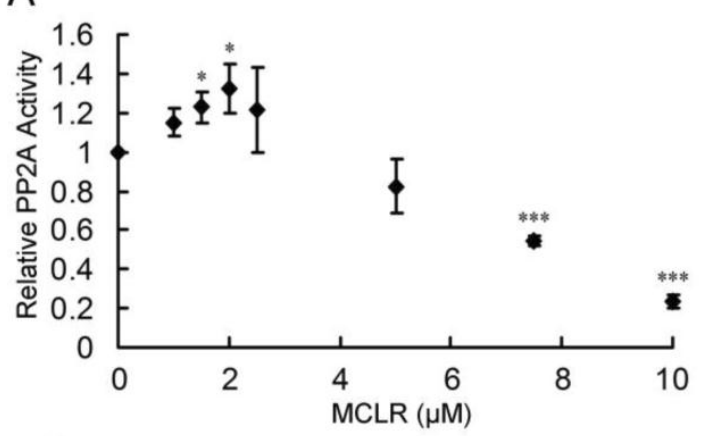

B

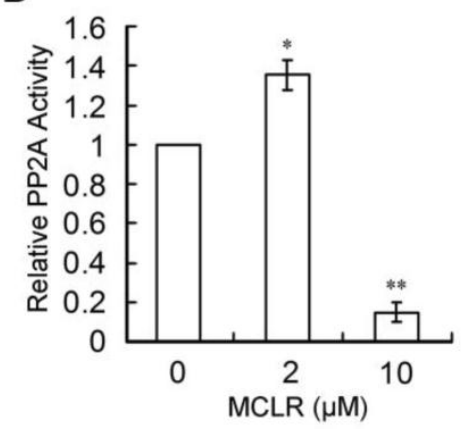

C

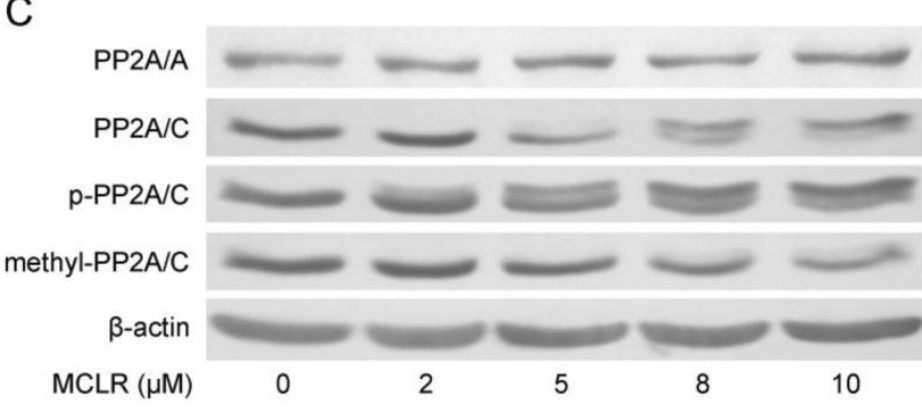

$\mathrm{D}$

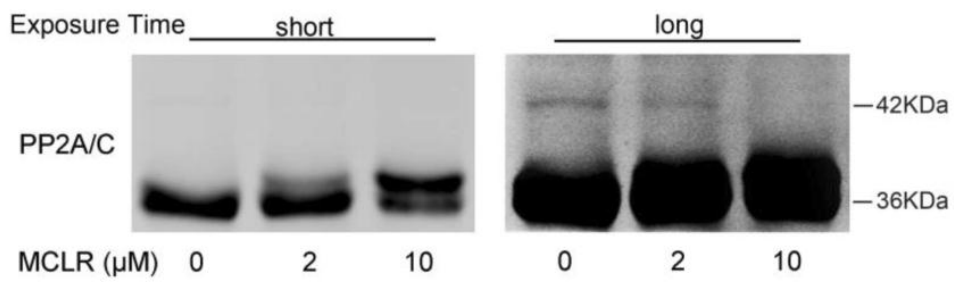

$\mathrm{E}$

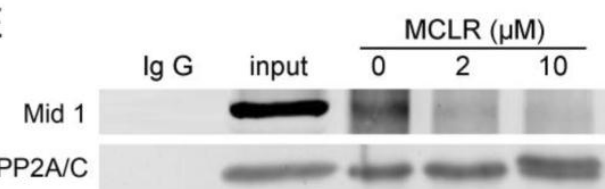

Figure 2. PP2A shows opposing activity with diffuse alternations of its core enzyme protein level and post-translational modification under MCLR treatment. HEK293 cells were exposed to varying concentrations of MCLR for $24 \mathrm{~h}$ before PP2A analysis. (A) PP2A activity was measured with a Promega serine/threonine phosphatase kit assay. Data represent means $\pm S D(n=3) *, p<0.05 ; * *, p<0.01 ; * * *, p<0.001$ compared with control. (B) PP2A activity was measured by PP2A immunoprecipitation phosphatase assay. Data represent means $\pm S D(n=3) *, p<0.05 ;{ }^{* *}, p<0.01$ compared with control. (C) PP2A core enzyme subunits A and C, including phosphorylated and methylated subunit C, were examined by Western blotting, where B-actin was used as loading control. (D) Ubiquitination of PP2A/C was captured by Western blotting with an antibody against PP2A/C at different exposure time (short: 1min, long: 10min). (E) Immunoprecipitation assay was applied in HEK293 cell lysate using an anti-PP2A/C antibody. The association of PP2A/C with Mid 1 form precipitated material was detected by Western blotting.

Finally, we tested the ubiquitination of PP2A/C after MCLR exposure, because ubiquitination of $\mathrm{PP} 2 \mathrm{~A} / \mathrm{C}$ has been predicted to be related with its decreased phosphatase activity [28]. We adjust the amount of loaded protein according to previous Western blotting assay to keep the total amount of PP2A/C approximately the same, as shown in shot-time exposure result (Fig. 2D left). At long-time exposure (Fig. 2D right), we captured a slight but ob- servable ladder of band, differing in molecular mass about $8 \mathrm{kDa}$ above the major band, which might suggest the monoubiquitination of PP2A/C [16-17]. Observably, this band decreased at $2 \mu \mathrm{M}$ and $10 \mu \mathrm{M}$ groups. Because ubiquitination of PP2A/C has been predicted to decrease PP2A activity, the loss of ubiquitination might suggest a possible correlation with the upregulation of PP2A activity at low dose of MCLR treatment. We further tried to reinforce the 
ubiquitination result using immunoprecipitation to test the interaction between PP2A/C and its E3 ubiquitin ligase Mid 1, which mediate the binding of $\mathrm{PP} 2 \mathrm{~A} / \mathrm{C}$ and ubiquitin. As shown in Fig. 2E, the binding of PP2A/C and Mid 1 decreased in both $2 \mu \mathrm{M}$ and $10 \mu \mathrm{M}$ groups, which is consistent with the previous result. Although the relationship between ubiquitination of PP2A/C and its activity is not clear yet, and our data might, therefore, only suggest a tenuous link between them, it gave us a hint to look into further evidence to explain the opposing PP2A activity change.

\section{a4 and PP2A C subunit dissociate under MCLR treatment}

Because the binding of PP2A/C and Mid 1 is reported to be managed by a special PP2A regulatory subunit, termed a4 [17], which is also an important PP2A activity regulator under cellular stress, we next focused our study to the interaction between PP2A/C and $a 4$. To address the role of $a 4$ in the regulation of PP2A in HEK293 cells with MCLR treatment, we at first examined the protein level of $a 4$ after $2 \mu \mathrm{M}-10$ $\mu \mathrm{M}$ MCLR exposure. As shown in Fig. 3A, a4 was constantly expressed across all concentrations. Next, the binding of $a 4$ and PP2A/C was measured by immunoprecipitation. As shown in Fig. 3B, the binding of $a 4$ and PP2A/C decreased in a MCLR concentration-dependent manner; about $60 \%$ of the original complex dissociated at $2 \mu \mathrm{M}$ and about $85 \%$ dissociated at $10 \mu \mathrm{M}$. The result confirmed the former PP2A ubiquitination assay and explained why PP2A activity is upregulated by a low concentration of MCLR: the PP2A/C released from a4 regains catalytic activity and thus compensate for the activity loss. The hypothesis was confirmed by probing PP2A/A from the immunoprecipitate. The interaction between PP2A A and $C$ subunits showed a trend of $10 \%$ up-regulation in the $2 \mu \mathrm{M}$ group. In the $10 \mu \mathrm{M}$ group, a remarkable dissociation of core enzyme (AC) was seen; only about $20 \%$ of PP2A/A remained bound to PP2A/C. The result is not only consistent with the drastic PP2A activity loss, but confirms the important function of $a 4$ in the assembly of the PP2A core enzyme, as recently reported [16]. The immunofluorescence assay was used to check the previous result by staining both $a 4$ and PP2A/C. Consistently, $a 4$ and PP2A/C showed visibly abated co-localization (Fig. 3C), as $a 4$ increasingly located at nuclei, while PP2A/C kept locating mainly at cytoplasm. We conclude from these data that $a 4$ and PP2A/C dissociate under MCLR exposure and may cause the opposite changes of PP2A activities at different MCLR concentrations.

\section{MCLR destabilized HEK293 cells cytoskeleton}

We next studied the cellular effect brought by the dissociation of $a 4$ and PP2A/C. Because $a 4$ is reported to be essential for maintaining the stability of cytoskeleton architecture, especially regarding cellular actin polymerization and focal adhesion formation [19], we investigated the polymerization of actin and morphology of vinculin, an important focal adhesion protein. HEK 293 cells were treated by $2 \mu \mathrm{M}$ or $10 \mu \mathrm{M}$ MCLR for 24 hours before immunofluorescence assay. As shown in Fig. 4A, decreased polymerization of actin was observed in $10 \mu \mathrm{M}$ group. Moreover in 10 $\mu \mathrm{M}$ group, vinculin localized in focal adhesion at cell extensions was decreased (Fig. 4B). While no obvious changes were observed in $2 \mu \mathrm{M}$ group. Given the knowledge that $a 4$ plays an important role in regulating actin polymerization and focal adhesion, these data might suggest that the aggressive dissociation of $a 4$ and PP2A/C might be related to the destabilization of cytoskeleton caused by MCLR.

\section{MCLR may induce anoikis in HEK293 cells}

Anoikis is a form of apoptosis induced by cell detachment from the extracellular matrix [29]. Because $a 4$ plays an essential role in opposing anoikis via PP2A, we next investigated the anoikis-related changes accompanied with $a 4 / \mathrm{PP} 2 \mathrm{~A} / \mathrm{C}$ dissociation after MCLR treatment. Photos were taken and detachment was measured by cell counting as described in Experimental Procedures (Fig. 5A, B). A trend of cell rounding was evident in the $10 \mu \mathrm{M}$ group. Compared with the control group, cells in $5 \mu \mathrm{M}$ and $10 \mu \mathrm{M}$ groups were detached by the wash of PBS in a dose-dependent manner, while the $2 \mu \mathrm{M}$ group showed nearly no additional detachment. In the MTT assay, we found the vitality of cells decreased accordingly to the detachment assay, as statistically positive vitality decrease was shown in $5 \mu \mathrm{M}$ and above concentration groups (Fig. 5B). Next, we measured several proteins important to anoikis or apoptosis (Fig. 6A). Apoptotic protein Bad upregulated its protein level, while anti-apoptotic protein Bcl-2 decreased its protein level at high concentration MCLR treatment; the full length of caspase-3 remained its protein level, while cleaved caspase- 3 increased in high concentration MCLR groups, as a hallmark of anoikis/apoptosis induced by MCLR [30]. The previous immunofluorescence result of decreased focal adhesion formation was also a symptom of anoikis [31]. We infer from these data that MCLR causes HEK293 cells detachment possibly further anoikis. 
A

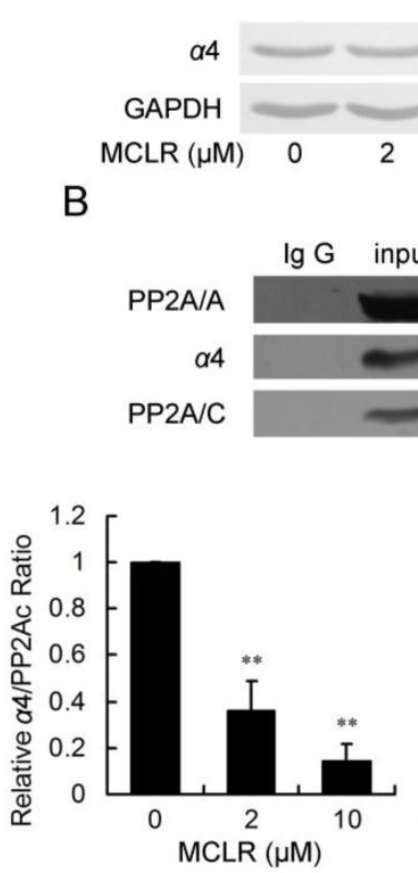

C

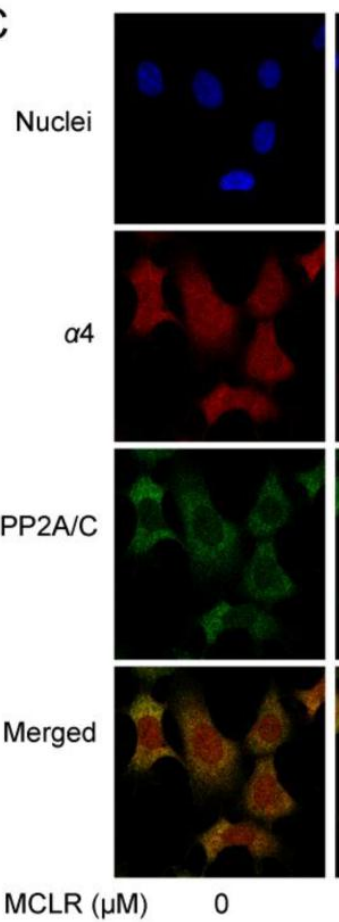

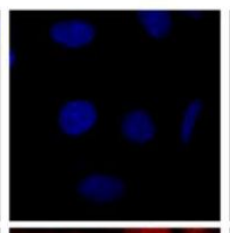
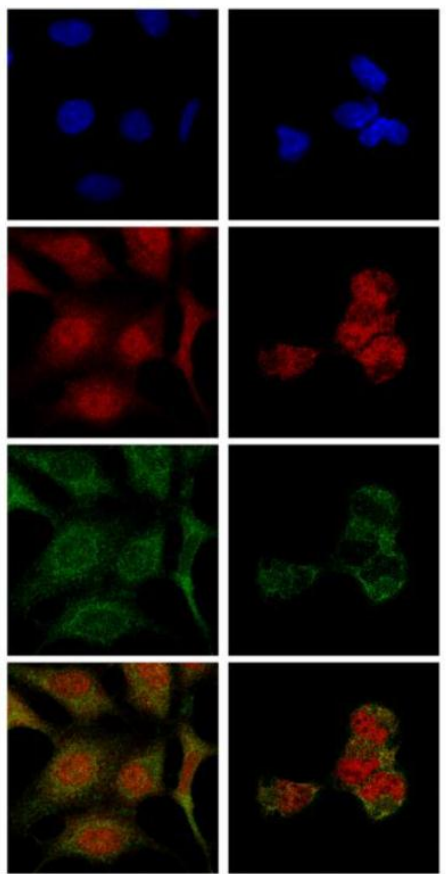

2

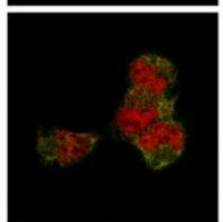

10

Figure 3. MCLR triggers a4/PP2A C subunit dissociation and affects PP2A core enzyme stability. HEK293 cells were treated with MCLR at varying concentrations for $24 \mathrm{~h}$. (A) The $a 4$ protein level was examined by Western blotting, where GAPDH was used as loading control. (B) Immunoprecipitation assay was performed in HEK293 cell lysate using an anti-PP2A/C antibody. The association of PP2A/C with a4 and PP2A/A form precipitated material was detected by Western blotting. Binding of PP2A/A or a4 to PP2A/C was quantified and the ratio of each complex was measured by comparing to total $C$ subunit and displayed by comparing to non-MCLR treated group. Data represent mean $\pm S D(n=3)$ ${ }^{*}, \mathrm{p}<0.05 ;{ }^{* * *}, \mathrm{p}<0.001$ compared with control. (C) Immunofluorescence assay using antibody against PP2A/C and $a 4$ shows reduced binding between $a 4$ (red) and PP2A/C (green). Nuclei (blue) were stained with DAPI. The merged images showed the co-localization of $a 4$ and PP2A/C.

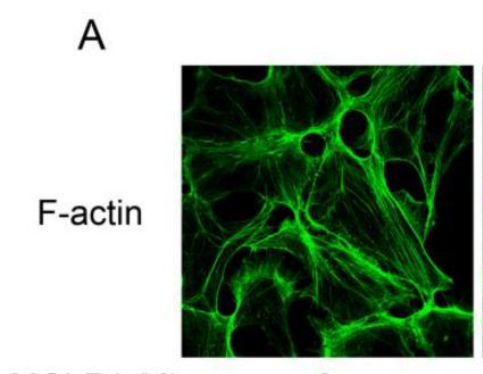

$\operatorname{MCLR}(\mu \mathrm{M})$

$\mathrm{B}$

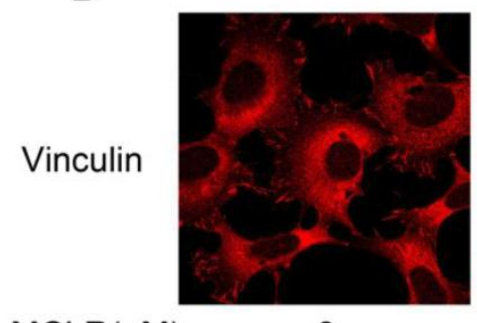

$\operatorname{MCLR}(\mu \mathrm{M})$

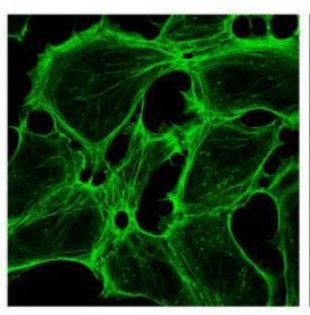

2

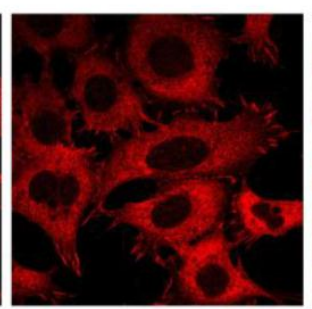

2

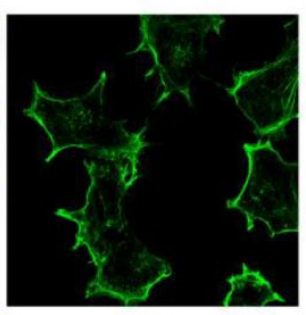

10

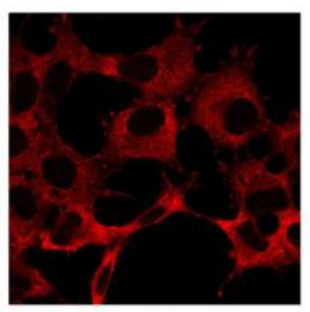

10

Figure 4. MCLR destabilize HEK293 cells cytoskeleton. HEK293 cells were treated with MCLR at varying concentrations for $24 \mathrm{~h}$ before immunofluorescence assays. (A) F-actin (green) was stained with phallotoxins. (B) Vinculin (red) was stained with antibody against vinculin. 
A
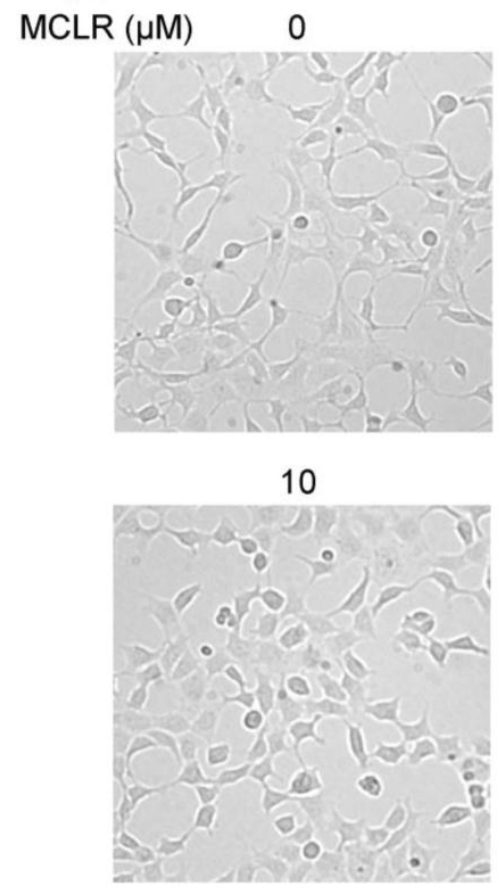

2

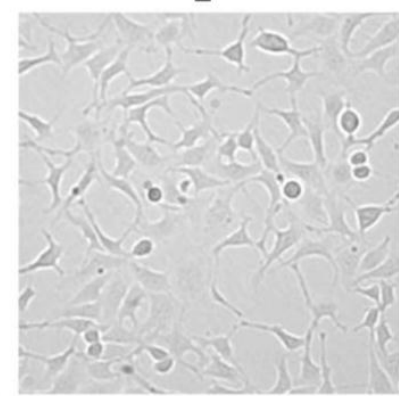

$\mathrm{B}$

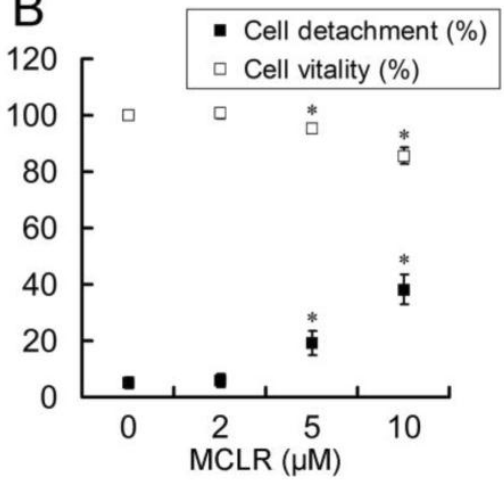

Figure 5. MCLR induces HEK293 cells morphology change, detachment and cell death. MCLR at varying concentrations was used to treat HEK293 cells for $24 \mathrm{~h}$. (A) Microscope photos of live cells. Representative micrographs show morphological change of HEK293 cells in the $10 \mu \mathrm{M}$ group. (B) Quantification of detachment and viability of HEK293 cells following MCLR disposure. Data represent mean $\pm S D(n=3){ }^{*}, p<0.05 ;{ }^{* *}, p<0.01 ;{ }^{* * *}, p<0.001$ compared with control.

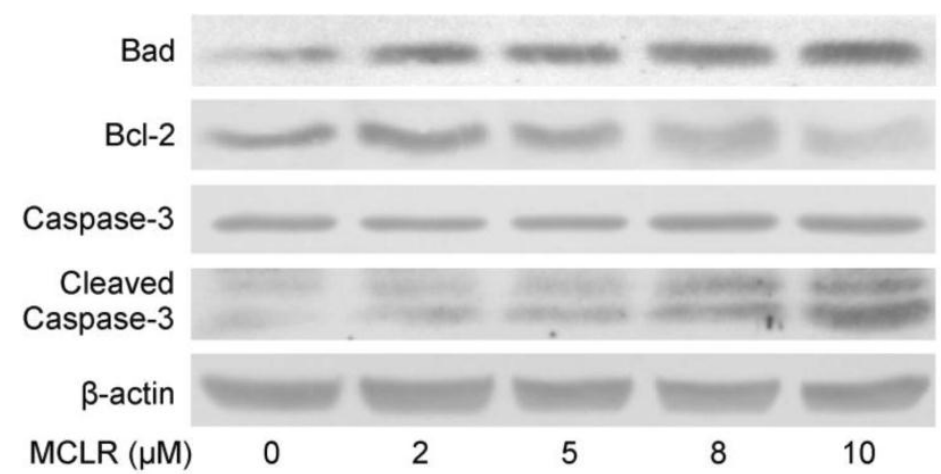

Figure 6. MCLR induces changes of anoikis-related proteins. (A) HEK293 cells were treated by MCLR for $24 \mathrm{~h}$ and lysed. Protein level of Bad, Bcl-2, full length caspase-3 (35KDA) and cleaved caspase-3 (17 and 19KDA) were examined by Western blotting, where $B$-actin was used as loading control. 


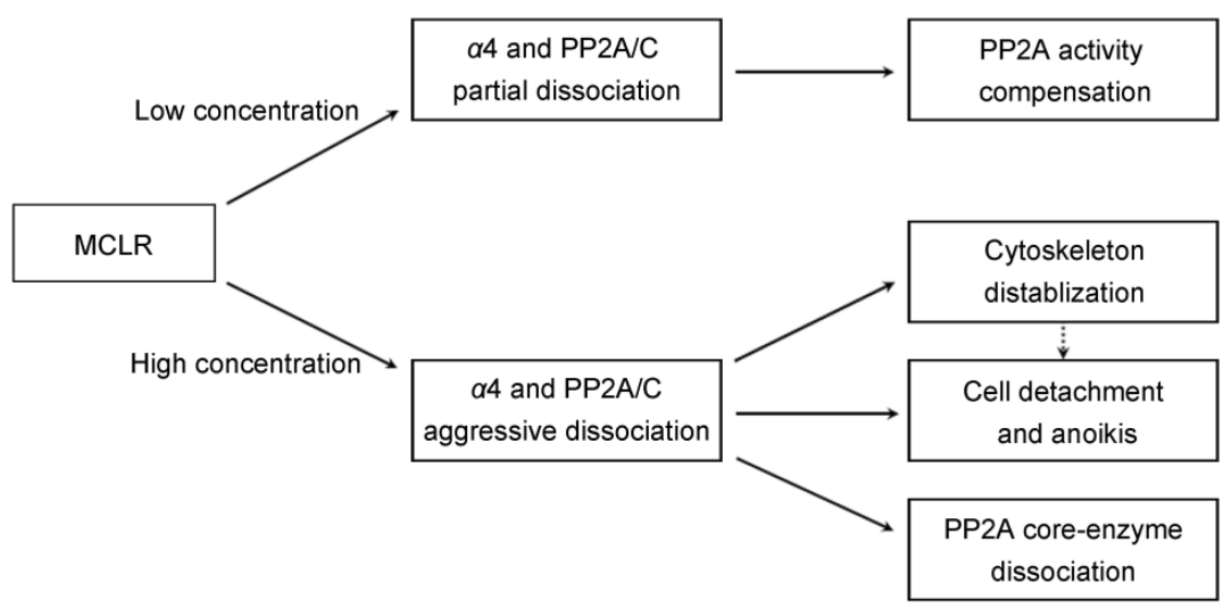

Figure 7. Schematic representation of multiple cellular effects induced by low and high concentration of MCLR through a4 and PP2A.

Table 1. Protein levels of different proteins after MCLR treatment. Densitometric analysis was performed by Image $\mathrm{J}$ software. Results were expressed by mean \pm SD from three independent experiments. The protein expression from control group was designated as 1 and the others were expressed as folds compared with the control. In case of multiple bands, the values represent all bands detected. The asterisk symbol represents statistical significance in comparison with the control value $\left({ }^{*}, p<0.05 ;{ }^{* *}, p<0.01\right)$.

\begin{tabular}{lllllll}
\hline & & \multicolumn{5}{c}{ MCLR $(\mu \mathrm{M})$} \\
\cline { 3 - 7 } & & \multicolumn{1}{c}{0} & \multicolumn{1}{c}{5} & \multicolumn{1}{c}{8} & 10 \\
\cline { 3 - 7 } Fig. 2 & PP2A/A & 1.00 & $0.89 \pm 0.11$ & $1.13 \pm 0.16$ & $0.87 \pm 0.17$ & $0.94 \pm 0.13$ \\
Fig. 2 & PP2A/C & 1.00 & $1.03 \pm 0.11$ & $0.96 \pm 0.29$ & $0.83 \pm 0.17$ & $0.80 \pm 0.19$ \\
Fig. 2 & p-PP2A/C & 1.00 & $0.97 \pm 0.14$ & $1.06 \pm 0.13$ & $1.31 \pm 0.20$ & $1.30 \pm 0.13$ \\
Fig. 2 & methyl-PP2A/C & 1.00 & $1.12 \pm 0.05$ & $0.66 \pm 0.10$ & $0.50 \pm 0.13^{*}$ & $0.28 \pm 0.07^{* *}$ \\
Fig. 3 & 44 & 1.00 & $0.97 \pm 0.10$ & $1.18 \pm 0.19$ & $0.98 \pm 0.36$ & $1.01 \pm 0.28$ \\
Fig. 6 & Bad & 1.00 & $1.60 \pm 0.36$ & $2.49 \pm 0.47^{*}$ & $2.84 \pm 0.44^{*}$ & $3.51 \pm 0.47^{*}$ \\
Fig. 6 & Bcl-2 & 1.00 & $1.24 \pm 0.28$ & $0.74 \pm 0.21$ & $0.55 \pm 0.16^{*}$ & $0.41 \pm 0.11^{*}$ \\
Fig. 6 & caspase 3 & 1.00 & $0.91 \pm 0.10$ & $0.90 \pm 0.11$ & $1.15 \pm 0.10$ & $1.17 \pm 0.18$ \\
Fig. 6 & cleaved caspase 3 & 1.00 & $0.98 \pm 0.19$ & $1.31 \pm 0.25$ & $1.91 \pm 0.35^{*}$ & $2.77 \pm 0.79^{*}$ \\
\hline
\end{tabular}

\section{Discussion}

The current study described multiple changes after MCLR treatment. Importantly, most of these effects are related to the function of $a 4$ and the dissociation of $a 4$ and PP2A/C. At low concentration treatment, the partial dissociation of $a 4$ and PP2A/C may results in the compensational upregulation of PP2A activity. At high concentration MCLR treatment, the aggressive dissociation of $a 4$ and PP2A/C may lead to the dissociation of PP2A core enzyme, destabilization of cytoskeleton, cell detachment and possibly further anoikis (Fig.7).

MCs, especially MCLR and MCRR, have been known and utilized as PP2A inhibitors for decades.
But the effect of MCs on PP2A in cell, especially at relatively low concentration, has seldom been studied. Our results, for the first time show that besides inhibition, MCLR at relatively low concentration can activate PP2A in cells. The nonlinear effect is characteristic of hormesis, a dose-response phenomenon of by low-dose stimulation and high-dose inhibition. It has recently been reported that MCLR at low concentration stimulates proliferation, while at relatively high concentration decrease cellular viability, in another kidney cell line, Vero-E6 cells. Such phenomenon might seemingly resemble hormesis, though the term was not mentioned or proposed by the authors [26, 32]. Still, whether MCLR could cause hormesis and if PP2A activity is a potential mark for hormesis 
detection are two questions needed to be further studied.

It is well established that the $a 4 / \mathrm{PP} 2 \mathrm{~A} / \mathrm{C}$ complex is inactive or has low catalytic activity [14-15]. In this study, the $a 4 / \mathrm{PP} 2 \mathrm{~A} / \mathrm{C}$ complex compensates the loss of PP2A by dissociation at relatively low concentration of MCLR. Kong and colleagues recently reported that $a 4$ is essential for PP2A core enzyme assembly and stability, and theorized that under cellular stress, the PP2A/C released from $a 4$ is required for the adaptive increase in PP2A activity [16]. Our PP2A activity assay and immunoprecipitation results demonstrate these two important functions of a4. Further, we provide evidence of the relationship between the two seemingly diffuse functions: the disruption of the binding between $a 4$ and $C$ subunit increases PP2A activity, possibly indicating that the released $\mathrm{C}$ subunit has shifted to the A subunit; on the other hand, $a 4$ is essential for the stable binding of the PP2A A and C subunit. So does the dissociation of the a4/PP2A/C complex enhance or destabilize the core enzyme? In our data, the relatively low MCLR concentration results in partial a4/PP2A/C dissociation, which may yield more core enzyme and increase PP2A activity. However, as the dissociation is aggravated by a relatively high MCLR concentration, the PP2A core enzyme is disrupted and facilitates drastic PP2A activity loss caused by MCLR. Therefore, we propose that partial dissociation of $a 4 / \mathrm{PP} 2 \mathrm{~A} / \mathrm{C}$ could result in an adaptive increase of PP2A activity, but as the dissociation continues and crosses a certain limit, the core enzyme of PP2A becomes unstable. Certainly, we could not rule out the possibility that MCLR binding to PP2A/C directly disrupts the core enzyme. But because $a 4$ is essential for core enzyme stability and assembly, it is more likely that MCLR affects PP2A core enzyme formation in this way.

It is reported that $a 4$ is important for maintaining cytoskeleton architecture and opposing anoikis/apoptosis [19-20]. In our data, aggressive dissociation of $a 4$ and PP2A/C at high concentration MCLR treatment also correlated with the destabilized cytoskeleton, cell detachment and possible anoikis. Given the major role $a 4$ played in these cellular processes, we propose the aggressive dissociation of a4 from PP2A/C renders its ability to maintain cytoskeletal stability and cell attachment, and further opposing anoikis; while partial dissociation may still preserve most of these functions, like the proposed model of PP2A activity regulation.

In addition to the dual effect on PP2A activity, we demonstrate in this study that MCLR affects the modification of PP2A/C protein. (i) The $1 \mathrm{kDa}$ up-shift of PP2A/C possibly suggests MCLR-conjunct band of
PP2A/C, which may also be related with the inhibition of PP2A activity. (ii) While the anti-p-PP2A/C antibody picks up both bands of PP2A/C, the relative strengths of the upper bands to lower bands observably increase with MCLR treatment. However, the anti-methyl-PP2A/C antibody nearly does not recognize the upper band, with only a very slight band at highest MCLR concentration group. Such phenomenon might suggest that phosphorylation of PP2A/C, rather than methylation of $\mathrm{PP} 2 \mathrm{~A} / \mathrm{C}$, favors MCLR-conjunct band. (iii) The increase of phosphorylated of PP2A/C is correlated with loss of PP2A activity. Our date might thereby, provide evidence for the cause-and-effect relationship between the two [33-34]. Since PP2A inhibition by MCLR is likely the more initial and direct cause of PP2A activity loss, our data might suggest that the loss of PP2A activity might render its ability to reactivate itself by autodephosphorylation, as reported [34].

The results of the present study suggest that MCLR triggers a compensation in PP2A activity; a former inactive complex of $a 4$ and PP2A/C dissociates upon exposure to MCLR, and the newly released PP2A/C may overturn the inhibition exerted by MCLR. However, as the inhibition rises with a higher MCLR concentration, the released PP2A/C is limited for the full recovery of PP2A activity loss, and PP2A activity decreases aggressively. Moreover, we find that the dissociation of $a 4$ and PP2A/C is correlated with cytoskeleton destabilization, cell detachment and possible anoikis, which are preserved or opposed by a4. Thus the multiple cellular changes may also indicate the consequences followed by the dissociation. Together, these results place $a 4$ not only in the heart of PP2A activity regulation but also in an overall cellular response encountering MCLR in HEK293 cells.

Biochemical analysis would require $a 4$ over-expression and knock-down to further illuminate the exact role of $a 4$ in regulating PP2A and other cellular processes under the exposure to MCLR. However, considering the potential toxicity of transfection, and the report that knock-down of a 4 causes direct apoptosis, we stick to genetically un-modified cells. Nevertheless, our results for the first time show that MCLR at low concentration can stimulate cellular PP2A activity, and further illustrate that a4 and $\mathrm{PP} 2 \mathrm{~A} / \mathrm{C}$ dissociation may be one reason, if not the only reason, contributed to this unexpected phenomena.

Recent studies of PP2A as a tumor suppressor have nudged it to the brink of being a potential target for cancer therapy [8, 35-36]. Increasing numbers of PP2A activators have been found, such as FTY720, forskolin, ceramide and D-erythro-S [37-38], and some 
of them have been proposed for drug development without actually knowing the activation mechanism [36]. By contrast, MCLR has long been ruled out of such a list because of its similar inhibition on both PP1 and PP2A activity (as shown in in vitro assay). Here with PP2A activation induced by its very own inhibitor MCLR and recently reported doxorubicin, it is possible that the in cell or in vivo influence of chemicals on PP2A may not be as simple as what it shows in vitro. So far as the activation of PP2A is concerned, the compensation mechanism provides a hint for the understanding of a general PP2A activation mechanism.

\section{Acknowledgements}

We thank Ning Li and Yajuan Guo from Professor Wei Liu's laboratory for expert technical assistance. This work was supported by the National Nature Science Foundation of China [No. 30771827 and No. 20777067] and the Key Special Program on the ST of China for the Pollution Control and Treatment of Water Bodies [No. 2008ZX07421-001].

\section{Conflict of Interests}

The authors have declared that no conflict of interest exists.

\section{References}

1. Codd GA, Morrison LF, Metcalf JS. Cyanobacterial toxins: risk management for health protection. Toxicol Appl Pharmacol. 2005;203:264-72.

2. MacKintosh C, Beattie KA, Klumpp S, Cohen P, Codd GA. Cyanobacterial microcystin-LR is a potent and specific inhibitor of protein phosphatases 1 and 2A from both mammals and higher plants. FEBS Lett. 1990;264:187-92.

3. Xing Y, Xu Y, Chen Y, Jeffrey PD, Chao Y, Lin Z, et al. Structure of protein phosphatase 2A core enzyme bound to tumor-inducing toxins. Cell. 2006;127:341-53.

4. Chen T, Wang Q, Cui J, Yang W, Shi Q, Hua Z, et al. Induction of apoptosis in mouse liver by microcystin-LR: a combined transcriptomic, proteomic, and simulation strategy. Mol Cell Proteomics. 2005;4:958-74.

5. Fu WY, Xu LH, Yu YN. Proteomic analysis of cellular response to microcystin in human amnion FL cells. J Proteome Res. 2005;4:2207-15.

6. Fu W, Yu Y, Xu L. Identification of temporal differentially expressed protein responses to microcystin in human amniotic epithelial cells. Chem Res Toxicol. 2009;22:41-51.

7. Janssens V, Goris J. Protein phosphatase 2A: a highly regulated family of serine/threonine phosphatases implicated in cell growth and signalling. Biochem J. 2001;353:417-39.

8. Eichhorn PJ, Creyghton MP, Bernards R. Protein phosphatase 2A regulatory subunits and cancer. Biochim Biophys Acta. 2009;1795:1-15

9. Van Hoof C, Goris J. Phosphatases in apoptosis: to be or not to be, PP2A is in the heart of the question. Biochim Biophys Acta. 2003;1640:97-104.

10. Cho US, $\mathrm{Xu} \mathrm{W}$. Crystal structure of a protein phosphatase $2 \mathrm{~A}$ heterotrimeric holoenzyme. Nature. 2007;445:53-7.
11. Xing ML, Wang XF, Xu LH. Alteration of proteins expression in apoptotic FL cells induced by MCLR. Environ Toxicol. 2008;23:451-8.

12. Liu J, Prickett TD, Elliott E, Meroni G, Brautigan DL. Phosphorylation and microtubule association of the Opitz syndrome protein mid-1 is regulated by protein phosphatase $2 \mathrm{~A}$ via binding to the regulatory subunit alpha 4 . Proc Natl Acad Sci U S A. 2001;98:6650-5.

13. Prickett TD, Brautigan DL. Overlapping binding sites in protein phosphatase $2 \mathrm{~A}$ for association with regulatory A and alpha- 4 (mTap42) subunits. J Biol Chem. 2004;279:38912-20.

14. Nanahoshi M, Tsujishita $Y$, Tokunaga C, Inui S, Sakaguchi N, Hara $K$, et al. Alpha4 protein as a common regulator of type 2A-related serine/threonine protein phosphatases. FEBS Lett. 1999;446:108-12.

15. Yoo SJ, Jimenez RH, Sanders JA, Boylan JM, Brautigan DL, Gruppuso PA. The alpha4-containing form of protein phosphatase 2A in liver and hepatic cells. J Cell Biochem. 2008;105:290-300.

16. Kong M, Ditsworth D, Lindsten T, Thompson CB. Alpha4 is an essential regulator of PP2A phosphatase activity. Mol Cell. 2009;36:51-60.

17. McConnell JL, Watkins GR, Soss SE, Franz HS, McCorvey LR, Spiller BW, et al. Alpha4 is a ubiquitin-binding protein that regulates protein serine/threonine phosphatase $2 \mathrm{~A}$ ubiquitination. Biochemistry. 2010;49:1713-8.

18. Prickett TD, Brautigan DL. Cytokine activation of p38 mitogen-activated protein kinase and apoptosis is opposed by alpha-4 targeting of protein phosphatase $2 \mathrm{~A}$ for site-specific dephosphorylation of MEK3. Mol Cell Biol. 2007;27:4217-27.

19. Kong M, Bui TV, Ditsworth D, Gruber JJ, Goncharov D, Krymskaya VP, et al. The PP2A-associated protein alpha4 plays a critical role in the regulation of cell spreading and migration. J Biol Chem. 2007;282:29712-20.

20. Kong M, Fox CJ, Mu J, Solt L, Xu A, Cinalli RM, et al. The PP2A-associated protein alpha4 is an essential inhibitor of apoptosis. Science. 2004;306:695-8.

21. Aranda-Orgilles B, Aigner J, Kunath M, Lurz R, Schneider R, Schweiger $S$. Active transport of the ubiquitin ligase MID1 along the microtubules is regulated by protein phosphatase $2 \mathrm{~A}$. PLoS One. 2008;3:e3507.

22. Bradford MM. A rapid and sensitive method for the quantitation of microgram quantities of protein utilizing the principle of protein-dye binding. Anal Biochem. 1976;72:248-54.

23. Widau RC, Jin Y, Dixon SA, Wadzinski BE, Gallagher PJ. Protein phosphatase 2A (PP2A) holoenzymes regulate death-associated protein kinase (DAPK) in ceramide-induced anoikis. J Biol Chem. 2010;285:13827-38.

24. Feurstein D, Holst K, Fischer A, Dietrich DR. Oatp-associated uptake and toxicity of microcystins in primary murine whole brain cells. Toxicol Appl Pharmacol. 2009;234:247-55.

25. Komatsu M, Furukawa T, Ikeda R, Takumi S, Nong Q, Aoyama $\mathrm{K}$, et al. Involvement of mitogen-activated protein kinase signaling pathways in microcystin-LR-induced apoptosis after its selective uptake mediated by OATP1B1 and OATP1B3. Toxicol Sci. 2007;97:407-16.

26. Dias E, Andrade M, Alverca E, Pereira P, Batoreu MC, Jordan P, et al. Comparative study of the cytotoxic effect of microcistin-LR and purified extracts from Microcystis aeruginosa on a kidney cell line. Toxicon. 2009;53:487-95.

27. Fu WY, Chen JP, Wang XM, Xu LH. Altered expression of p53, Bcl-2 and Bax induced by microcystin-LR in vivo and in vitro. Toxicon. 2005;46:171-7.

28. Trockenbacher A, Suckow V, Foerster J, Winter J, Krauss S, Ropers HH, et al. MID1, mutated in Opitz syndrome, encodes an ubiquitin ligase that targets phosphatase $2 \mathrm{~A}$ for degradation. Nat Genet. 2001;29:287-94. 
29. Frisch SM, Francis H. Disruption of epithelial cell-matrix interactions induces apoptosis. J Cell Biol. 1994;124:619-26.

30. Fladmark KE, Brustugun OT, Hovland R, Boe R, Gjertsen BT, Zhivotovsky B, et al. Ultrarapid caspase-3 dependent apoptosis induction by serine/threonine phosphatase inhibitors. Cell Death Differ. 1999;6(11):1099-108.

31. Gilmore AP. Anoikis. Cell Death Differ. 2005;12 (Suppl 2):1473-7.

32. Dias E, Matos $P$, Pereira $P$, Batoreu MC, Silva MJ, Jordan P. Microcystin-LR activates the ERK1/2 kinases and stimulates the proliferation of the monkey kidney-derived cell line Vero-E6. Toxicol In Vitro. 2010;24:1689-95.

33. Guo H, Damuni Z. Autophosphorylation-activated protein kinase phosphorylates and inactivates protein phosphatase 2A. Proc Natl Acad Sci U S A. 1993;90:2500-4.

34. Chen J, Martin BL, Brautigan DL. Regulation of protein serine-threonine phosphatase type-2A by tyrosine phosphorylation. Science. 1992;257:1261-4.

35. Mumby M. PP2A: unveiling a reluctant tumor suppressor. Cell. 2007;130:21-4.

36. McConnell JL, Wadzinski BE. Targeting protein serine/threonine phosphatases for drug development. Mol Pharmacol. 2009;75:1249-61.

37. Dobrowsky RT, Kamibayashi C, Mumby MC, Hannun YA. Ceramide activates heterotrimeric protein phosphatase 2A. J Biol Chem. 1993;268:15523-30.

38. Mukhopadhyay A, Saddoughi SA, Song P, Sultan I, Ponnusamy S, Senkal CE, et al. Direct interaction between the inhibitor 2 and ceramide via sphingolipid-protein binding is involved in the regulation of protein phosphatase 2A activity and signaling. FASEB J. 2009;23:751-63.

\section{Figures}

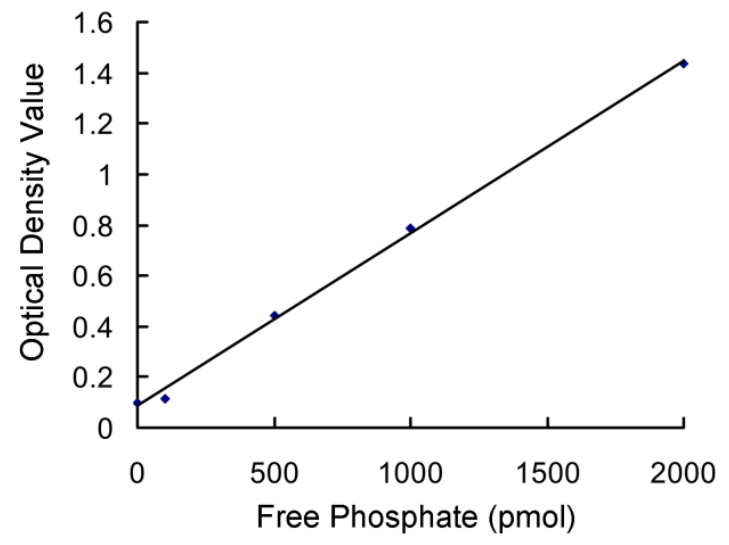

Figure S1. Standard Curve from PP2A activity assays. Different amount of free phosphate standard from Promega serine/threonine phosphatase assay system were diluted and measured in 96-well plate, for the use of standard curve. 